\title{
Disseminating widely
}

To the Editor - There is certainly a need for technical guidance on materials characterization and a harmonized approach for the scientific community as suggested in your Editorial 'Join the dialogue' (Nature Nanotech. 7, 545; 2012). However, given the potential impact of nanomaterials on human health, technological progress and economic benefits, we think that other stakeholders such as regulators, decision makers, members of the innovation community and industrial partners also need to be informed on important science-based issues surrounding the safety of nanomaterials and technologies. Disseminating messages more widely will possibly increase the importance of nanosafety on the societal and policy agenda.
To reach these stakeholders, it is necessary to identify key topical issues on nanosafety and condense them into short, easy-to-read messages. For example, the Finnish Institute of Occupational Health (FIOH) distributes 'FIOH Impact Sheets' in Finnish a few times a year to Finnish decision makers. When justified by the FIOH strategy and goals, the top leadership decides ad hoc on the release of these documents considering the general interest and timeliness of the topic. These sheets are distributed in English to decision makers globally when the topic they address is of a global nature and interest. This is a new practice at the FIOH; the impact sheet on nanotechnology is the third that has been distributed in Finnish, and the first that has been distributed in English. The most recent impact sheet was published on 31 October 2012 and was entitled 'Safety Research on Nanotechnology Needed' (http://go.nature. com/f5kok6). It highlights the importance of the safety of workers, consumers and the environment to gain trust and confidence on engineered nanomaterials, and to promote innovation and commercialization of nanomaterials globally. We believe that this condensed and easy-to-read format of a policy brief for domestic and international decision makers is a valuable and efficient tool to increase awareness.

Kai Savolainen ${ }^{\star}$ and Harri Alenius

Finnish Institute of Occupational Health, Helsinki 00250, Finland.

*e-mail: kai.savolainen@ttl.fi

\section{Regulatory and research needs}

To the Editor - Parallel to your Editorial 'Join the dialogue' (Nature Nanotech. 7, 545; 2012), the Hazardous Substances Advisory Committee, which advises the UK government, also considered the subject of reporting standards for nanotoxicological research and has published its findings online (http://go.nature.com/wxNgtl). We highlight the key findings here.

Implementing consistent standards is broader than simply reporting appropriate physical and chemical characterization; it starts with problem formulation and hypothesis generation. At its broadest level, procurement of nanomaterials and their characterization can be differentiated for regulatory and research purposes. For regulatory purposes, the standards applied and data generation required must be more prescriptive, whereas for research these must be primarily based on the hypothesis to be tested.

However, minimum requirements in both areas include the reason for the choice of the nanomaterials, appropriate sample pre-treatment, physical and chemical characterization, and measurement of actual dose to which an organism is exposed. Sample pre-treatment must be considered because, for instance, the use of traditional high-vacuum microscopy and spectroscopy is inappropriate for hydrated and organic-rich samples; analysis can change the conformation, aggregation state and other nanomaterial properties. Characterization must also be performed using an appropriate multi-method approach to obtain unbiased measurements. For example, the indiscriminate use of dynamic light scattering to measure the size of polydisperse, aggregated and nonspherical samples should be discouraged because the data produced are inaccurate. Electron microscopy should be made quantitative and additional detectors should be used to extract useful parameters such as shape, elemental composition and chemistry from a sufficient number of randomly chosen images. Dose, which is the concentration of toxicant to which an organism or cell is exposed, is a fundamental parameter in toxicology. Because dynamic changes (such as sorptive losses to container walls, aggregation or dissolution) can occur during exposure of nanomaterials to cells, the use of nominal (added) dose is dubious. Dose and characterization, therefore, must be measured over the appropriate exposure time and conditions. The details of and data from metrology and characterization should be fully reported in any published paper. Without such information the comparability and utility of biological hazard data is highly questionable.

Jamie Lead ${ }^{1 \star}$ and Stephen Holgate ${ }^{2}$

${ }^{1}$ Arnold School of Public Health, University of South Carolina, Columbia, South Carolina 29208, USA, ${ }^{2}$ School of Medicine, University of Southampton, Mailpoint 810, Level F, Southampton SO16 6YD, UK. *e-mail: JLEAD@mailbox.sc.edu

\section{Complexities abound}

To the Editor - Your September 2012 Editorial ${ }^{1}$ pointed out the lack of materials characterization and poor reporting as the main causes of inconsistency in the nanobio interaction literature. Although this is true, the Editorial did not fully identify the complexities of the research involved.

Although there have been advances in characterization techniques, less has been done to identify a universal descriptive nomenclature system for nanomaterials; it is expected that well-characterized nanomaterials and a naming system would enable researchers to compare biological results. A 'nanomaterials classification 